\title{
The moderating role of population succession in the adaptive responses of Synechococcus assemblages: evidence from light intensity simulation experiment
}

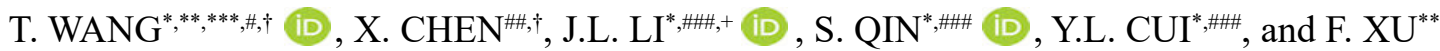

Key Laboratory of Coastal Biology and Biological Resource Utilization, Yantai Institute of Coastal Zone Research, Chinese Academy of Sciences, 264000 Yantai, China*

College of Environmental Science and Engineering, Ocean University of China, 266100 Qingdao, China ${ }^{* *}$

CAS Key Laboratory for Experimental Study under Deep-sea Extreme Conditions, Institute of Deep-sea Science and Engineering, Chinese Academy of Sciences, 572000 Sanya, China ${ }^{* * *}$

University of Chinese Academy of Sciences, Beijing, China

College of Marine Life Science, Ocean University of China, 266005 Qingdao, China ${ }^{\# \#}$

Center for Ocean Mega-Science, Chinese Academy of Sciences, 266071 Qingdao, China

\begin{abstract}
Synechococcus is one of the most abundant photoautotrophic picoplankton in the marine ecosystem. However, it is not clear how Synechococcus assemblages respond to light intensity variation in a genus group. Here, enriched Synechococcus assemblages from in situ coastal seawater were subjected to light intensity simulation experiments in a range of 9-243 $\mu \mathrm{mol}$ (photon) $\mathrm{m}^{-2} \mathrm{~s}^{-1}$. Characteristics concerning physiology, genomics, and metatranscriptomics were analyzed. Physiologically, the fitting model predicted photosynthesis indications and pigment contents increased with different trends following the light intensity. Genomic sequencing demonstrated that both the phylogenetic and phenotypic compositions of Synechococcus assemblage exhibited population succession. Especially, the proportion of Synechococcus pigment type 2 was changed significantly. In metatranscriptomics, most genes were downregulated in the high-light intensity group, while photosynthesis-related genes were entirely upregulated. The high upregulation of photosynthesis-related genes, such as $p s b O, p s b A, a p c B$, and $c p c B$, corresponded to the succession of Synechococcus genotype and was responsible for the physiological shift in response to light intensity.
\end{abstract}

Keywords: enrichment culture; genomics; irradiance; metatranscriptomics; picocyanobacteria.

\section{Highlights}

- Both phylogenetic and phenotypic compositions of Synechococcus showed alterations

- Pigment contents and photosynthesis indicators increased with light intensity at different trends

- Regulation of photosynthetic genes was associated with the succession of genotypes
Received 21 July 2021

Accepted 25 October 2021

Published online 18 November 2021

${ }^{+}$Corresponding author

e-mail: j1li@yic.ac.cn

Abbreviations: ANOVA - analysis of variance; $\mathrm{AP}$ - allophycocyanin; CA - carbonic anhydrase; Chl - chlorophyll; DEGs - differentially expressed genes; FDR - false discovery rate; GO - Gene Ontology; KEGG - Kyoto Encyclopedia of Genes and Genomes; LI - light intensity; LSM - least-square method; OTU - operational taxonomic unit; PE - phycoerythrin; PEC - phycoerythrocyanin; PC - phycocyanin; PCR - polymerase chain reaction; PT - pigment type; RDA - redundancy analysis; VIF - variance inflation factor; WGCNA - weighted gene co-expression network analysis.

Acknowledgements: This study was financially supported by the Key Deployment Project of Centre for Ocean Mega-Science, Chinese Academy of Sciences (COMS2020Q09), the National Key Research and Development Program of China (2018YFD0901102), and the Science and Technology Program of Yantai (2017ZH090 and 2017ZH095). We extend our gratitude to the journal reviewers for their comments and suggestions, which helped in significantly improving the manuscript.

${ }^{\dagger}$ Co-first authors.

Conflict of interest: The authors declare that they have no conflict of interest. 


\section{Introduction}

Marine Synechococcus, a polyphyletic group of picoplanktonic cyanobacteria, is one of the most abundant photoautotrophs in the oceans worldwide (Sohm et al. 2016). As an important marine picoplankton with global distribution, Synechococcus plays a significant role in the carbon flux and primary production in oceanic waters (Guidi et al. 2016). Estimates show that Synechococcus contributes $8 \mathrm{Gt}$ of $\mathrm{C}$ to the net primary production per year, which is responsible for $16.7 \%$ of the marine net primary production, exceeding the production of other phytoplankton species (Flombaum et al. 2013).

In response to global climate change, the distribution of Synechococcus has changed considerably both in spatial and vertical scales. For example, a massive northward shift of species was observed with an increase in temperature. Indeed, Synechococcus has been recorded far beyond the polar circle (Paulsen et al. 2016); an expansion of its dominant area in the marine ecosystem is predicted (Flombaum et al. 2013). Light is considered an effective factor that determines the vertical distribution of Synechococcus in natural water bodies (Everroad and Wood 2012). The turbidity caused by suspended particulate matters, which has increased with an intensification of human activities (van der Hout et al. 2017), and the occurrence of algal blooms, such as the red tide due to eutrophication (Zohdi and Abbaspour 2019), have altered the light environment, including both light quality and light intensity (LI). Studies have shown that the abundance of Synechococcus is maximum in the surface mixed layer and declines with depth (Rocap et al. 2003, Scanlan et al. 2009). Synechococcus is typically less abundant below $100 \mathrm{~m}$; however, it is found at depths of 200-600 m and even deeper during deep mixing events (Lindell and Post 1995, DuRand et al. 2001). The high physiological plasticity of Synechococcus indicates that its distribution may increase in the future. Hence, investigation of deep adaptation mechanisms in response to changes in environmental conditions is required.

Studies regarding the photosynthetic adaptive strategies of phytoplankton are important in the field of marine biology and physiology (Halsey and Jones 2015). Until now, researchers have conducted a series of in situ studies or pure culture-based physiological analyses to understand the intrinsic adaptation mechanisms in response to LI variation. Field measurements of chlorophyll fluorescence revealed that picophytoplankton in highlight and low-nutrient open sea preserved PSII activity by diverting electrons from the PSI complex-mediated carbon assimilation to oxygen (Mackey et al. 2008). Meanwhile, it maintained high photosynthetic efficiency and simultaneously prevent photodamage via a propyl gallate-sensitive oxidase associated with the photosynthetic electron transport chain. Marine Synechococcus lineages have also evolved ecotype differentiation in response to light, as observed in isolated culture experiments and clone library data (Ahlgren and Rocap 2006). Furthermore, proteomic research on light responses, ranging from optimized low light to optimized high light, revealed that the proteins of Synechococcus strains involved in photosynthetic electron transport, carbon fixation, and oxidative stress protection (superoxide dismutases) varied. The unique combinations of light responses in different strains resulted in distinct photophysiological phenotypes that may result in Synechococcus niche division in the ocean (Mackey et al. 2017).

Culture experiments based on purified Synechococcus isolates are the most common methods used for investigating the adaptation mechanisms of phytoplankton under different environmental stresses (Śliwińska-Wilczewska et al. 2020). However, Synechococcus is a genus with rich phylogenetic and phenotypic diversity, and pure cultures of only rare strains have been successfully established so far (Grébert et al. 2018, Xia et al. 2019). In addition, the succession of community and reciprocal or competitive interactions between strains are neglected to a considerable extent in pure culture.

Hence, in this study, we conducted a controlled simulation experiment to analyze response to LI using enriched culture samples of Synechococcus obtained from in situ coastal water and investigated the variation characteristics at physiological, genomics, and transcriptomics levels. The specific aims are as follows: (1) to detect the variation in physiological parameters, including growth rate, photosynthetic efficiency, and pigment content of Synechococcus assemblages in the LIcontrolled experiment; (2) to determine the phylogenetic and phenogenetic community successions from in situ coastal water to enriched culture under LI gradient; (3) to explore the mechanisms underlying gene regulation of Synechococcus group in response to LI variation; (4) to construct the relationship among physiology, genomic structure, and gene regulation of Synechococcus assemblages for LI adaption.

\section{Materials and methods}

Sampling: Field sampling was conducted in the BHB10 station of the Bohai Bay on Research Vessel Kexue III on 24 August 2018 (Fig. 1S, supplement). Bohai Bay, a semi-closed bay, is located in the mid-latitude monsoon area. Its geohydrological conditions, along with the large amounts of pollutants discharged by the inflowing rivers, make it one of the most polluted coastal areas in China (Gao and Chen 2012). The BHB10 station is located at the center of the Bohai Bay, which is adjacent to the Tianjin Port that experiences frequent occurrence of harmful algal blooms (Liu et al. 2019). Two parallel original seawaters, $\mathrm{B} 1$ and B2, were collected at BHB10. To detect the in situ composition of Synechococcus assemblages, 1,000 $\mathrm{ml}$ of seawater was filtered on $0.22-\mu \mathrm{m}$ polycarbonate filters (Millipore Co., Bedford, MA, USA). Triplicate membranes from each sample were placed in sterile cryopreservation tubes and flash-frozen in liquid nitrogen. Upon returning to the laboratory, the samples were stored at $-80^{\circ} \mathrm{C}$ for subsequent molecular experiments. For the simulation experiment, $100 \mathrm{ml}$ of seawater was collected in a sterile 
culture bottle containing $50 \mathrm{ml}$ of SNAX medium with actinomycin solution (final concentration $0.05 \mathrm{mg} \mathrm{ml}^{-1}$ ) to exclude eukaryotes (Deng et al. 2014, Hunter-Cevera et al. 2016). After returning to the laboratory, the samples were enrich-cultured for subsequent light-controlled experiments.

Culture experiments: The two enriched culture samples, B1 and B2, were inoculated with the same initial cell density in conical flasks containing the SNAX medium. The simulation experiment was performed in $Z Q Z Y-B G F 8$ combined light shaker incubators (Shanghai Zhichu Instruments Co., Ltd., China). The four LI were set as low (L), middle-low (ML), middle-high (MH), and high (H) LI gradients using 9, 27, 81, and $243 \mu \mathrm{mol}$ (photon) $\mathrm{m}^{-2} \mathrm{~s}^{-1}$, respectively, in three parallel samples. The light and dark cycle was set at 12:12 $\mathrm{h}$ and the temperature was maintained at $21^{\circ} \mathrm{C}$. Physiological parameters were determined after cultivating for $15 \mathrm{~d}$. All samples were collected in the end using $0.22 \mu \mathrm{m}$-polycarbonate membranes (Millipore Co., Bedford, MA, USA) for genomic and transcriptomic analysis.

Physiological parameters: During experimental periods, $1.40 \mathrm{ml}$ of culture liquid of each flask was taken in a sterile tube every day, followed by the addition of polyoxymethylene (the final concentration was $0.5 \%$ ) for recording cell density using flow cytometry (Nowack et al. 2015). The growth rate of each sample was calculated over the experimental period from the daily cell density using linear regression models (Jodłowska and Śliwińska 2014). The net photosynthetic rate (net oxygen growth rate) was monitored using an oxygen electrode (Hansatech, King's Lynn, UK) (Ueno et al. 2020). Carbonic anhydrase (CA, EC 4.2.1.1) activity was determined from the time required for the $\mathrm{pH}$ to decrease from 8.1 to 7.3 after ultrasonic extraction in $20 \mathrm{mM}$ barbiturate buffered saline (Hu et al. 2010). Chlorophyll (Chl) a content was determined from the optical densities at 647 and $664 \mathrm{~nm}$ after extraction with 90\% acetone (Wagner et al. 2016). Contents of phycobiliproteins, including phycoerythrin (PE), phycocyanin (PC), and allophycocyanin (AP), were determined from the optical densities at 565, 620, and $650 \mathrm{~nm}$ (UV-VIS spectrophotometer TU-1810, Persee, China), respectively, after ultrasonic extraction in $100 \mathrm{mM}$ phosphate-buffered saline (Mittal et al. 2017).

Genome sequencing and analysis: DNA was extracted from the filter membranes using the Fast DNA spin kit (MP BIO, USA) according to the manufacturer's protocol. Polymerase chain reaction (PCR)-mediated amplification of DNA was performed using the thermocycler GeneAmp ${ }^{\circledR}$ PCR system 9700 (Perkin Elmer, USA) in triplicate following previous methods (Li et al. 2019). rpoC1 was amplified using the primer pair rpoC1-39F (5-GGNATNGTNTGYGAGCGYTG-3) and rpoC1-462R (5-CGYAGRCGCTTGRTCAGCTT-3) (Xia et al. 2019). Then, the purified rpoC1 gene products were sequenced at the Majorbio Co., Ltd. (Shanghai, China) using the PE300 Illumina MiSeq sequencing platform. The cpeBA operon sequences were amplified using the primer pair peBF (5-GACCTACATCGCWCTGGGYG-3) and peAR (5-CCMACAACCARGCAGTAGTT-3) (Xia et al. 2018). The purified products of the $c p e B A$ operon were sequenced at the Shanghai Biozeron Biological Technology Co., Ltd. (Shanghai, China) using the PE250 Illumina MiSeq sequencing platform.

Raw FASTQ data were quality-controlled, paired, chimera-filtered, and redundancy eliminated for downstream analysis (Caporaso et al. 2010). Operational taxonomic unit (OTU) clustering was performed on the nonrepetitive sequences considering $97 \%$ similarity and the representative OTU sequences were obtained. The representative sequence of each OTU was identified using BLAST search (Altschul et al. 1997). Sequences that do not belong to the phylum Cyanobacteria were deleted from the results of MegaBlast. The most similar reference sequences were retrieved from the NCBI database [reference sequence information of rpoC1 is listed in Table 1S (supplement), and that of the cpeBA operon is listed in Table 2S (supplement)]. The sequence that accounted for more than $1 \%$ in each sample was aligned with reference sequences using ClustalW according to codon structure, and the Maximum Likelihood phylogenetic tree was constructed with $M E G A 7$ using the best model GTR $+\mathrm{G}+\mathrm{I}$ for nucleotide evolution with 200 bootstraps (Xia et al. 2018). Phylogenetic and phenotypic information of Synechococcus strains was derived from published literature (Grébert et al. 2018) and the Roscoff Culture Collection (https://roscoff-culturecollection.org/strains/shortlists/taxonomic-groups/marinesynechococcus).

Metatranscriptomic sequencing and analysis: Metatranscriptome analysis was performed to investigate the effect of LI on gene regulation. The physiological parameters of Synechococcus assemblage showed a nonlinear increase from 9 to $243 \mu \mathrm{mol}$ (photon) $\mathrm{m}^{-2} \mathrm{~s}^{-1}$; hence, we used the samples cultured under $\mathrm{L}$ and $\mathrm{H}$ for analysis. Total RNA was extracted from the filtered membranes using TRIzol ${ }^{\circledR}$ reagent according to the manufacturer's instructions and genomic DNA was removed using DNase I (TaKara). Then, rRNA was removed using the Ribo-Zero rRNA removal kits per the manufacturer's instructions (Illumina). Optimized high-quality RNA samples were used for downstream experiments. Metatranscriptome libraries were prepared using TruSeq ${ }^{T M}$ RNA sample preparation kit from Illumina (San Diego, CA, USA). Metatranscriptome sequencing was performed by Shanghai Biozeron Biotechnology Co., Ltd. using the Illumina Novaseq 6000 instrument.

The raw paired-end reads were trimmed and qualitycontrolled using Trimmomatic v. 0.36 (Bolger et al. 2014). High-quality reads were then aligned to the SILVA SSU $(16 \mathrm{~S} / 18 \mathrm{~S})$ and SILVA LSU (23S/28S) databases to remove rRNA-related reads using the SortMeRNA software (Kopylova et al. 2012). Then, clean data from all samples were used for assembly with MegaHit (Li et al. 2016). All the genes were predicted using METAProdigal (Hyatt et al. 2012). The nonredundant gene catalog was 
constructed with $95 \%$ identity and $90 \%$ coverage using CD-HIT (Fu et al. 2012). To identify the proteins that showed the highest sequence similarity with the given transcripts and retrieve their functional annotations, all genes were searched against the $N C B I$ protein nonredundant, String, and Kyoto Encyclopedia of Genes and Genomes $(K E G G)$ databases using BLASTp with E-values less than $1.0 \times 10^{-5}$. Metabolic pathway analysis was performed using KEGG (Kanehisa and Goto 2000). Salmon was used for calculating the expression level of each transcript, and RSEM was used to quantify gene and isoform abundance (Li and Dewey 2011, Roberts and Pachter 2013). EdgeR in $R$ was utilized for differential expression analysis (Robinson et al. 2010). The differentially expressed genes (DEGs) with logarithmic fold change in expression $>2$ and false discovery rate (FDR) $<0.05$ were selected for Gene Ontology $(G O)$ functional enrichment and KEGG pathway analysis (Klopfenstein et al. 2018). In total, 678 genes, the expression level of which was $\geq 10$ in at least one sample, were selected to perform weighted gene co-expression network analysis (WGCNA) using an $R$ package (Langfelder and Horvath 2008). Gephi and Cytoscape were used to visualize results from the co-expression networks. Hubba genes of each module were determined using Clusterine Coefficient of CytoHubba, a Cytoscape plugin (Chin et al. 2014).

Statistical analysis: Package basicTrendline in $R$ was used for fitting each physiological parameter with the corresponding LI using three models: linear, logarithmic curve, and power curve (Mei et al. 2018). Package agricolae in $R$ was used for one-way analysis of variance (ANOVA) with post hoc test (LSD) (de Mendiburu 2014). Package vegan in $R$ was used for calculating redundancy analysis (RDA) between physiological parameters and community composition (Oksanen et al. 2013). The leastsquare method (LSM) was used to further calculate the linear relationship between physiological parameters and the percentage of OTU. Packages psych and pheatmap in $R$ were used for sketching correlation heat map between gene expression as well as physiological parameters and Synechococcus composition.

Data availability: The sequence data of this study were deposited in the sequence read archive of $N C B I$. A bio-project associated with this study was applied for and processed in NCBI with the accession number PRJNA680823. All raw sequencing data were stored under this bio-project.

\section{Results}

Physiological responses to $\mathbf{L I}$ : The physiological responses of $\mathrm{B} 1$ and $\mathrm{B} 2$ to $\mathrm{LI}$ showed similar trends (Fig. 2S, supplement). All parameters increased with LI. The highest growth rate, net photosynthetic rate, CA activity, $\mathrm{PC}$ content, and AP content all appeared in $\mathrm{B} 2-\mathrm{H}$, reaching $0.45 \mathrm{~d}^{-1}, 1.42 \mathrm{nmol} \mathrm{l}^{-1} \mathrm{~s}^{-1}, 1.81 \times 10^{-2} \mathrm{EU} \mathrm{ml}^{-1}$, $3.77 \times 10^{-2} \mathrm{mg} \mathrm{ml}^{-1}$, and $1.45 \times 10^{-2} \mathrm{mg} \mathrm{ml}^{-1}$, respectively. The Chl $a$ and PE contents were maximal in B1-H, which were $1.06 \times 10^{-3} \mathrm{mg} \mathrm{ml}^{-1}$ and $1.12 \times 10^{-2} \mathrm{mg} \mathrm{ml}^{-1}$, respectively. Most $\mathrm{PE}, \mathrm{PC}$, and $\mathrm{AP}$ contents under $\mathrm{L}$ were below the detection limit. In B1, the CA activity under $\mathrm{MH}$ $\left(1.64 \times 10^{-2} \mathrm{EU} \mathrm{ml}^{-1}\right)$ was slightly higher than that under $\mathrm{H}$ $\left(1.58 \times 10^{-2} \mathrm{EU} \mathrm{ml}^{-1}\right)$ but did not differ significantly.

The nonlinear power curve model was always the best model for fitting each parameter with its LI (Table 3S, supplement). The power values, $b$, were less than 0 in models of growth rate, net photosynthetic rate, and CA activity; hence, the maximal value of these physiological parameters was dependent on the constant $c$ (Fig. 1). The values of $b$ in models of $\mathrm{Chl} a, \mathrm{PE}, \mathrm{PC}$, and $\mathrm{AP}$ content were between 0 and 1 , and consequently, these physiological parameters continued to increase with LI without attaining any maximal value. The $p$-value of each model was less than 0.01 , indicating the dependability of this nonlinear relationship between each parameter and LI.

Changes in genomic composition in response to $L I$ : On average, rpoC1 generated 21174 sequences with coverage of more than 0.99 in each sample (Table $4 \mathrm{~S}$, supplement). In total, 16 OTUs were detected with relative abundances accounting for more than $1 \%$ in each sample, among which 11 OTUs were from in situ samples, which decreased to 5 OTUs in cultured samples (Fig. $2 A$ ). These OTUs occupied $97.8 \%$ of the total sequences on average. All three Synechococcus subtypes, S5.1, S5.2, and S5.3 were detected, of which S5.1 was further divided into two clades: VIII and IX. Both samples of in situ seawater, B1-I and B2-I, were dominated by S5.1 clade IX, followed by S5.3 and S5.2. Clade VIII was the least abundant in the in situ water samples. Compared to those of the in situ samples, the Chao index and Shannon index of the cultured samples decreased to 86.3 and $85.4 \%$, respectively. The Synechococcus assemblage in all culture samples was composed of two OTUs of clade VIII, which contributed to $87.4 \%(\mathrm{~B} 1-\mathrm{MH})$ and $99.9 \%(\mathrm{~B} 2-\mathrm{H})$ sequence abundance. OTU 2380, most dominant in B2-MH and all culture samples of B1, occupied more than $82.8 \%$ of the sequences. OTU31 was the most abundant in $\mathrm{B} 2-\mathrm{H}$, reaching $97.5 \%$ abundance. In the remaining samples B2-ML and B2-L, each of these two OTUs accounted for about half of the sequences.

The cpeBA operon generated 40,825 sequences on average, with coverage of more than 0.99 in each sample (Table 4S). Thirty-three OTUs were detected using the cpeBA operon, accounting for more than $1 \%$ in each sample, among which 22 OTUs were from in situ samples; this decreased to 11 OTUs in the cultured samples (Fig. 2B), which occupied $82.90 \%$ of total sequences on average and included three PTs: PT2, PT3a, and PT3c/PT3d. A similar trend in both in situ and cultured samples was observed the proportion of PT2 was higher than that of PT3a and PT3c/PT3d. Compared to those of the in situ samples, the Chao index and Shannon index of the cultured samples decreased to 28.9 and $41.8 \%$, respectively. The proportion of PT2 increased two-fold in the samples after culture. In general, OTU1 (30.4-40.1\%) and OTU2 (32.2-40.0\%) accounted for a large bulk of the Synechococcus assemblage composition, while the other OTUs were rarely detected. 

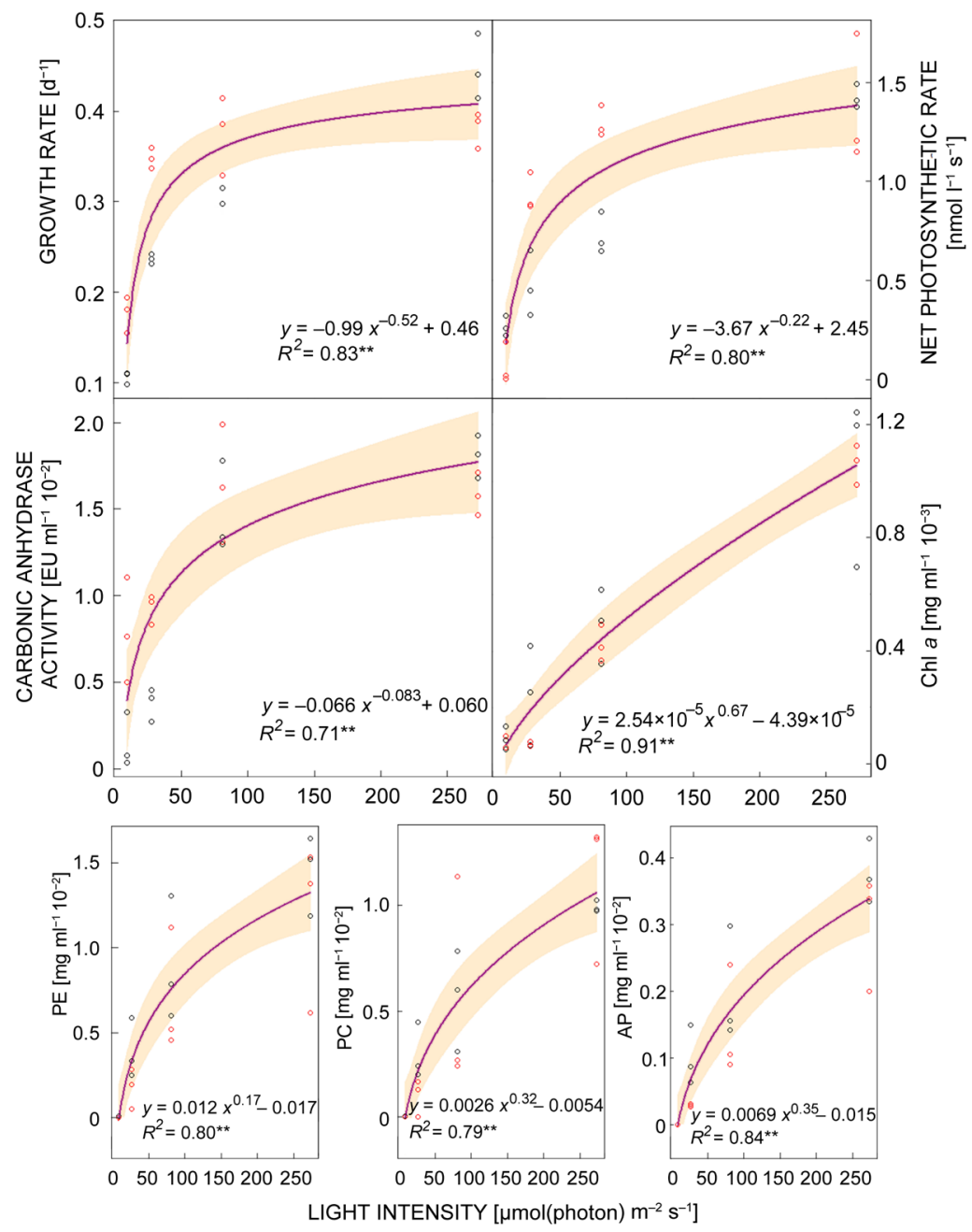

Fig. 1. Scatter plots with established power curve models of each physiological parameter with light intensity. Red circles represent the parameters measured in $\mathrm{B} 1$, while black circles represent that in $\mathrm{B} 2 .^{* *}-p<0.01$. PE - phycoerythrin; $\mathrm{PC}-$ phycocyanin; AP - allophycocyanin.

Significant differences in PT2 percentage between B1-MH, BI-ML, and B2-L were observed (Fig. 2D).

The VIF value indicated strong autocorrelation between contents of $\mathrm{PE}, \mathrm{PC}$, and $\mathrm{AP}$, leaving only $\mathrm{PC}$ for eliminating the effect of collinearity in calculating RDA. The permutation test for all constrained eigenvalues in the model of RDA between physiological parameters and OTUs based on rpoC1 showed statistical significance (Fig. 3A). Three physiological parameters, net photosynthetic rate $\left(R^{2}=0.34, p<0.01\right)$, Chl $a$ content $\left(R^{2}=0.37, p<0.01\right)$, and PC content $\left(R^{2}=0.24, p<0.05\right)$, significantly correlated with the community. Noticeably, the relationship between two main OTUs, OTU31 and OTU2380, and the parameters were opposite. Further fitting calculation showed a significant linear relationship between the percentage of OTU31 and PC content (Fig. 3S, supplement). However, relationships between most of the other OTUs and physiological parameters were not simply linear. Comparably, the permutation test for all constrained eigenvalues showed that the $p$-value of the RDA model between physiological parameters and OTUs based on cpeBA operon was 0.14 , which was $>0.05$, and hence did not reach statistical significance (Fig. $3 B$ ). Therefore, Spearman's correlation was calculated to investigate the interaction between phenotypes of Synechococcus and physiological parameters (Fig. 4S, supplement). We found that OTU2, OTU3, OTU6, and OTU8, which belonged to different PTs, showed significant correlations with all physiological parameters. OTU15 and OTU54 correlated with Chl $a$ content. Notably, the relationship between OTUs belonging to the same PT and physiological parameters could differ considerably.

Metatranscriptional response to $L I$ : The metatranscriptome sequencing of each sample generated about $20 \mathrm{~GB}$ of data. In total, 7,145 genes were differentially expressed between the $\mathrm{H}$ group (combined $\mathrm{B} 1-\mathrm{H}$ and B2-H) and the L group (combined B1-L and B2-L). Among them, the expression of 1,839 genes was significantly more in group $\mathrm{H}$ than in group L (hereafter, upregulated), and 5,306 genes were expressed significantly less in group $\mathrm{H}$ than in the $\mathrm{L}$ group (hereafter, downregulated) (Fig. 4A). In total, 3,252 differentially expressed genes were annotated in $81 K E G G$ pathways, which could be classified into six functions of $K E G G$ level 1 and 34 functions of $K E G G$ level 2 (Fig. 4B). Among the 50 genes with the highest expression level, eight genes were related to photosynthesis, and all of them were 

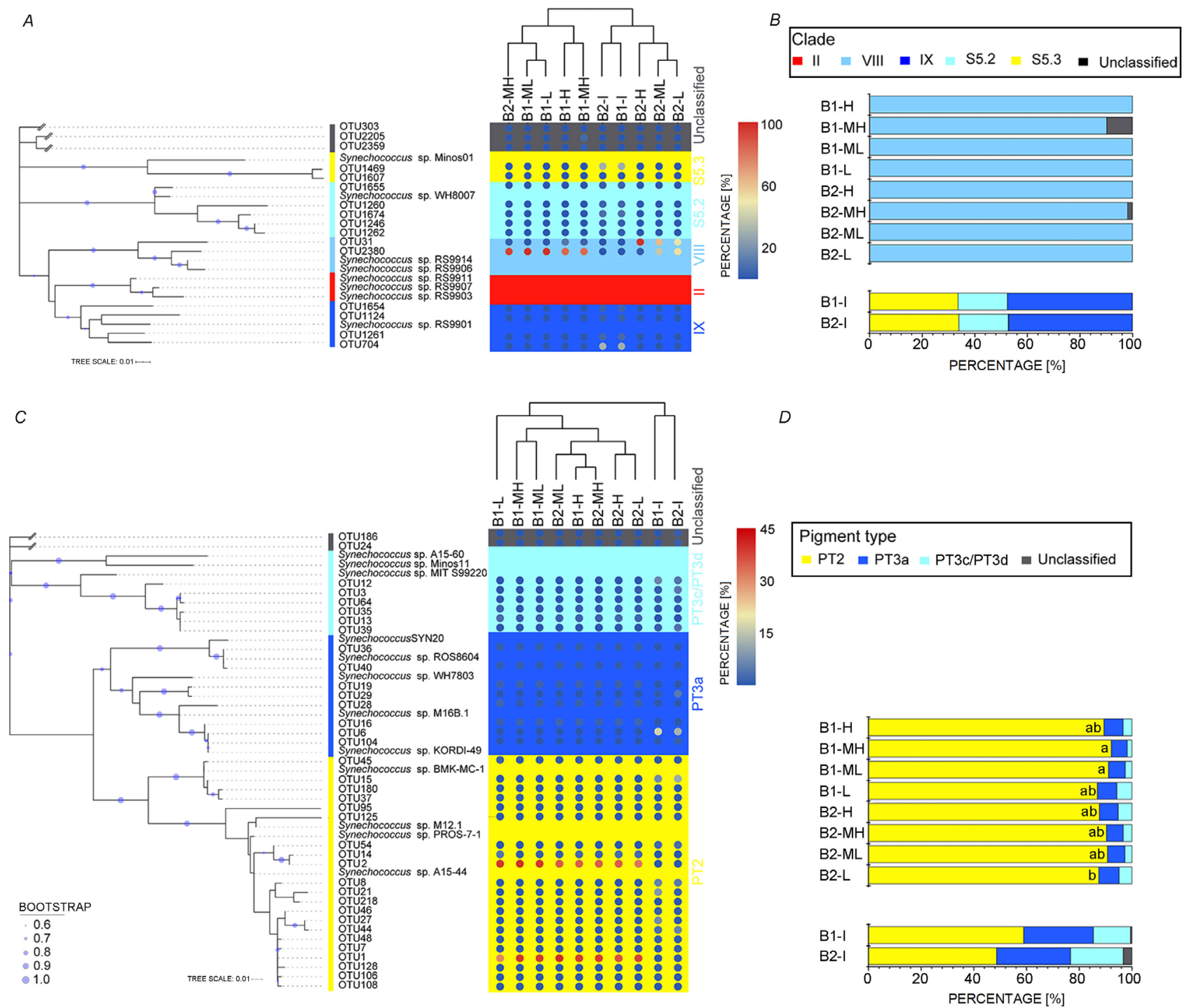

$D$
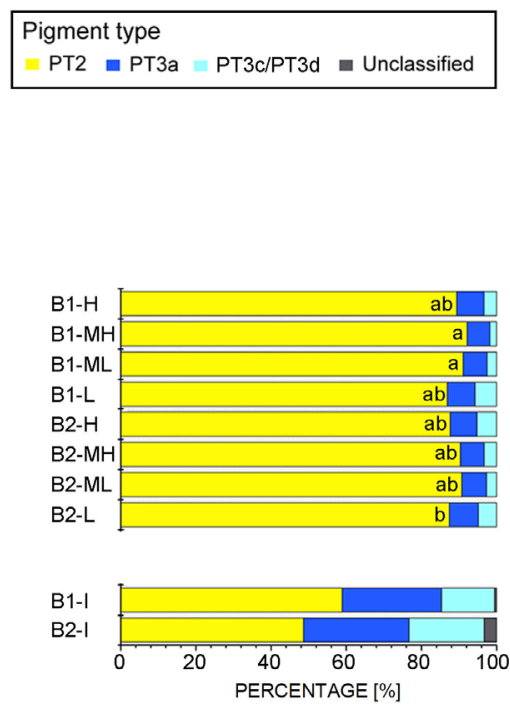

Fig. 2. (A) Maximum likelihood phylogenetic tree of operational taxonomic units (OTUs) accounting for more than $1 \%$ abundance based on rpoC1 sequence; the color of the circles in heatmaps shows the relative abundance of OTUs in each library with color legends on the right. (B) Stacking diagram showing the relative abundance of Synechococcus clades. (C) Maximum likelihood phylogenetic tree of OTUs accounting for more than $1 \%$ based on cpeBA operon; the color of the circles in heatmaps shows the relative abundance of OTUs in each library with color legends on the right. $(D)$ Stacking diagram showing the relative abundance of Synechococcus pigment type. ' $\backslash$ ' indicates collapsed tree branches that are far from the main tree. The lowercase letters in the stacking diagram represent the significant difference between samples calculated using one-way ANOVA with a post hoc test (LSD). B1-H - B1-high light intensity; B1-MH - B1-middle-high light intensity; B1-ML - B1-middle-low light intensity; B1-L - B1-low light intensity; B2-H - B2-high light intensity; B2-MH - B2-middle-high light intensity; B2-ML - B2-middle-low light intensity; B2-L - B2-low light intensity; B1-I B1-in situ; B2-I - B2-in situ.

upregulated in group H (Fig. 4C).

The genes associated with photosynthesis were mostly classified in two KEGG pathways, ko00195 and ko00196 (Fig. 5). One hundred and twenty-three genes were annotated in the pathway map of ko00195 with 27 unique functions, involving PSI, PSII, cytochrome $b_{6} f$ complex, photosynthetic electron transport, and F-type ATPase. Forty-five of these genes were upregulated and 78 were downregulated. In addition to the genes encoding the F-type ATPase, all other matched genes were upregulated.
Sixteen genes were annotated in the pathway map of ko00196 with 12 unique functions, involving regulation of AP, PC/phycoerythrocyanin (PEC), and PE; all these genes were upregulated.

Five significant Spearman correlations between gene expression associated with photosynthesis and physiological parameters were detected (Fig. 6A). All the correlations were strongly negative $\left(R^{2}<0.6\right)$. The expression levels of $p s a B$, psbO, and $p s b V$ were significantly associated with percentages of OTU31 and 


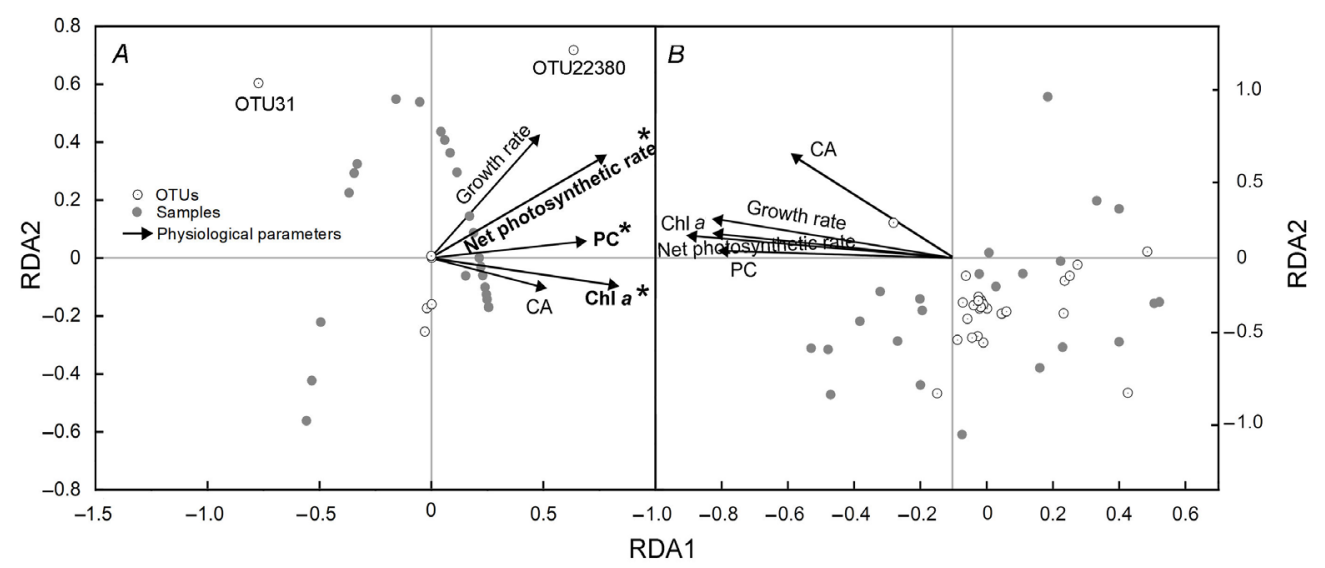

Fig. 3. Correlation plots based on a redundancy analysis (RDA) reveal the relationship between samples, physiological parameters, and operational taxonomic units accounting for more than $1 \%$ abundance based on $r p o \mathrm{Cl}(A)$ and $c p e \mathrm{BA}$ operon $(B) .{ }^{*}-p<0.05,{ }^{* *}-p<0.01$.
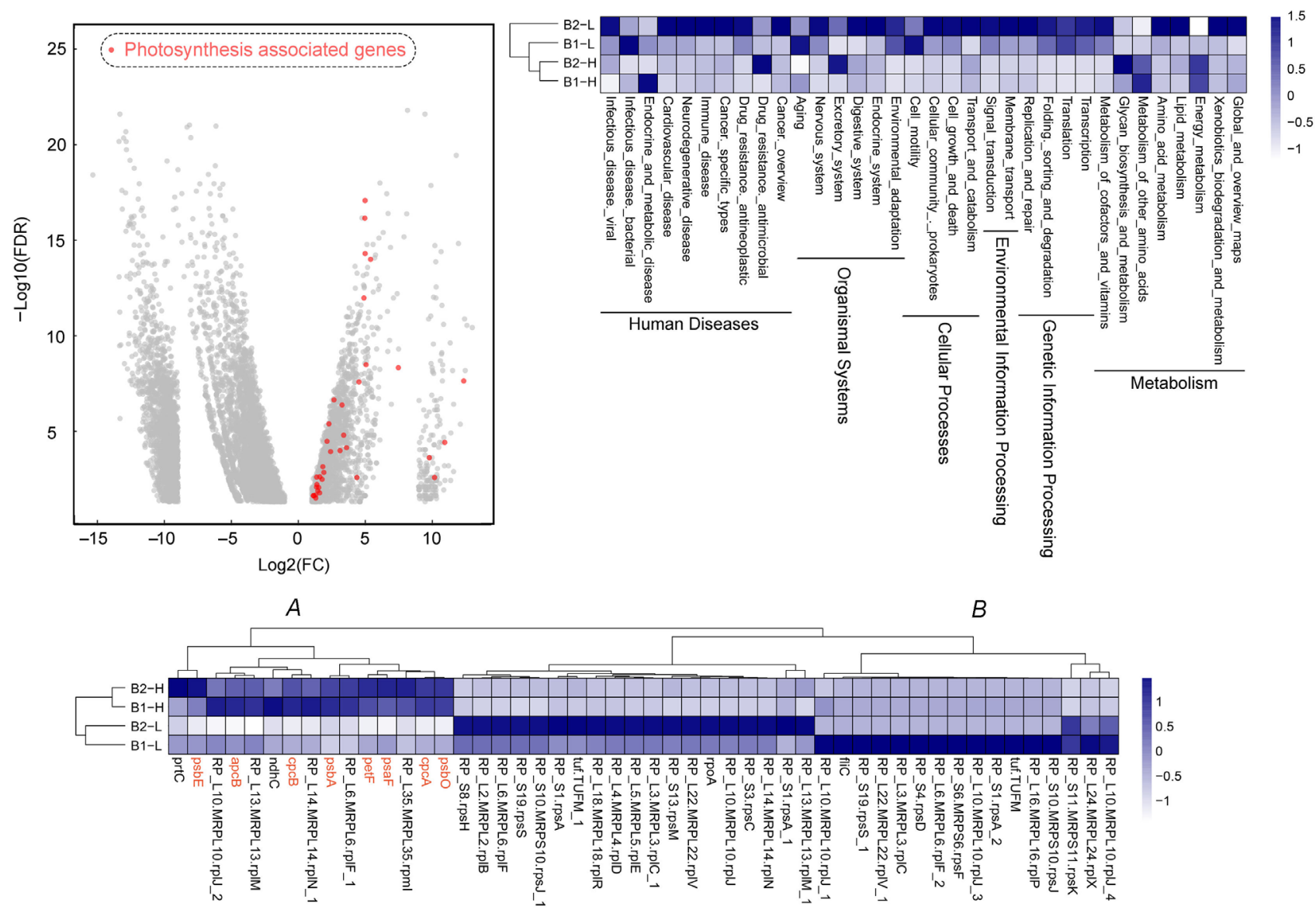

C

Fig. 4. (A) Volcano plots of the genes identified using metatranscriptomics [H (high-light intensity) group vs. L (low-light intensity) group]; the red dots represent genes associated with photosynthesis and the grey dots represent other differentially expressed genes. (B) Heat map of the gene expression at KEGG levels 1 and 2 based on metatranscriptomic sequencing. (C) Heat map of the 50 most expressive differential genes; genes associated with photosynthesis were marked in red. Data in heat maps were scaled and normalized. B1-H - B1-high light intensity; B1-L - B1-low light intensity; B2-H - B2-high light intensity; B2-L - B2-low light intensity.

OTU2380 (both OTUs belong to Synechococcus clade $\mathrm{VIII}$ ) in the sequence of rpoC1 (Fig. $6 B$ ). The expression of
13 types of genes correlated with the percentages of three OTUs, OTU108, OTU218, and OTU36 in the sequence 


\section{ko00195}

Photosystem II \begin{tabular}{|lllll}
$\mathrm{D} 1$ & $\mathrm{D} 2$ & $\mathrm{cp} 43$ & $\mathrm{cp} 47$ & cytb559 \\
\hline
\end{tabular}

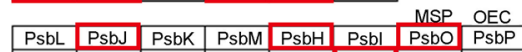

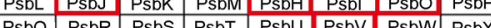
\begin{tabular}{l|l|l|l|l}
$P S b Q$ & PsbR & PsbS & PsbT & PsbU \\
\hline PsbY & PsbZ & Psb27 & Psb28 & Psb282 \\
\hline
\end{tabular}

Photosystem I

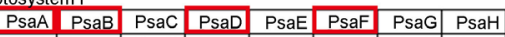

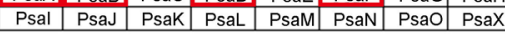

Cytochrome b6/f complex \begin{tabular}{|l|l|l|l|l|l|l|l|}
\hline PetB & PetD & PetA & PetC & PetL & PetM & PetN & PetG \\
\hline
\end{tabular}

Photosynthethic electron transport

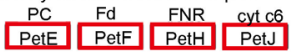

F-type ATPase

\begin{tabular}{|l|l|l|l|l|}
\hline beta alpha gamma delta epsilon & c & a & b \\
\hline
\end{tabular}

\section{ko00196}

Allophycocyanin (AP)

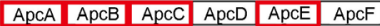

Phycocyanin (PC) / Phycoerythrocyanin (PEC)

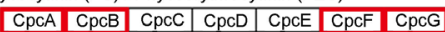

Phycoerythrin (PE)

\begin{tabular}{|l|l|l|l|l|l|l|l|}
\hline CpeA & CpeB & CpeC & CpeD & CpeE & CpeR & CpeS & CpeT \\
\hline
\end{tabular}

\begin{tabular}{|l|l|l|l|}
\hline CpeU & CpeY & CpeZ \\
\hline
\end{tabular}
Fig. 5. Pathway maps of entry ko00195 and ko00196 associated with photosynthesis annotated using the $K E G G$ database. The red box marked genes significantly upregulated in group $\mathrm{H}$ (high-light intensity) compared with those in group L (low-light intensity). The yellow box represents the presence of both upregulated and downregulated genes.

A

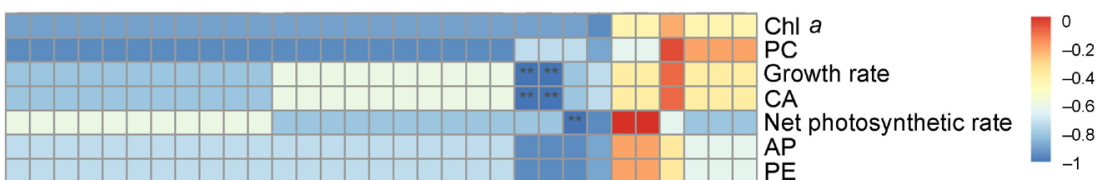

$B$
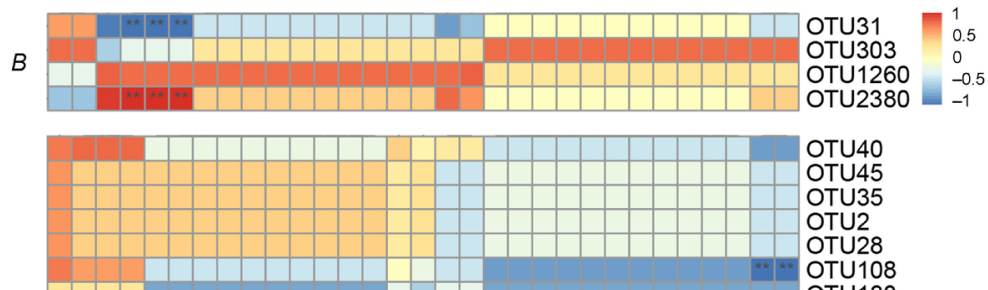

OTU40

OTU45

OTU35

OTU2

OTU28

OTU108
OTU180
OTU218

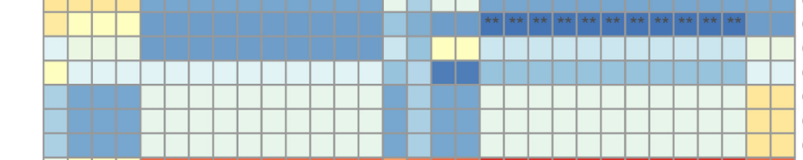

OTU1

OTU7

OTU95

OTU21

C

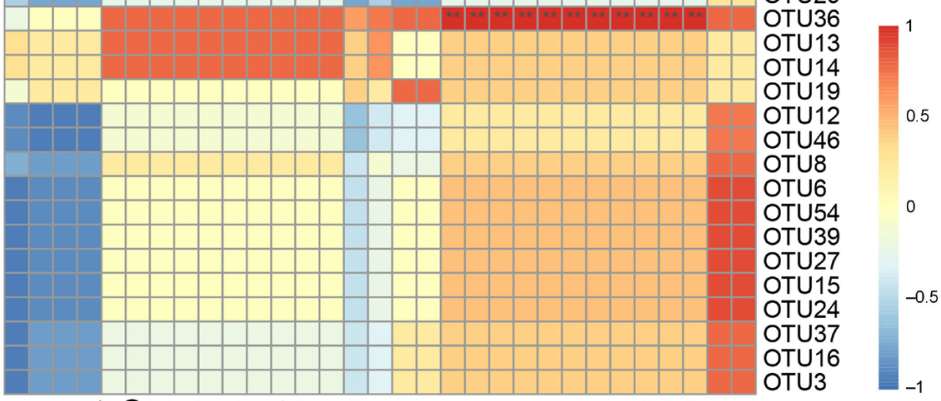

Fig. 6. Spearman correlation heat map between photosynthesis associated gene expression and physiological parameters $(A)$, operational taxonomic units (OTUs) accounting for more than $1 \%$ abundance based on rpoC1 $(B)$ and cpeBA operon (C). The color legends on the right represent the value of the Spearman correlation coefficient. ${ }^{*}-p<0.05,{ }^{* *}-p<0.01$. AP - allophycocyanin; CA carbonic anhydrase; PC - phycocyanin; PE phycoerythrin.

of the cpeBA operon (Fig. 6C). OTU108 and OTU218 belonged to Synechococcus PT2, while OTU36 belonged to Synechococcus PT3a.

Gene co-expression network analysis: Seven gene modules were identified from the results of WGCNA (Fig. 7; Fig. 5SA, supplement). Among them, modules 1, 5, and 6 clustered together based on the module eigengenes of modules and were separated from others (Fig. 5SB). The topological feature of co-expression networks constructed based on each module is listed in Table 5S (supplement). Module 7 occupied more than $44 \%$ of 678 genes with expression level $\geq 10$ in at least one sample. Modules 1 and 2 accounted for 27 and 16\% genes, respectively, and the other four modules added up to account for $13 \%$ genes. After annotation using the 

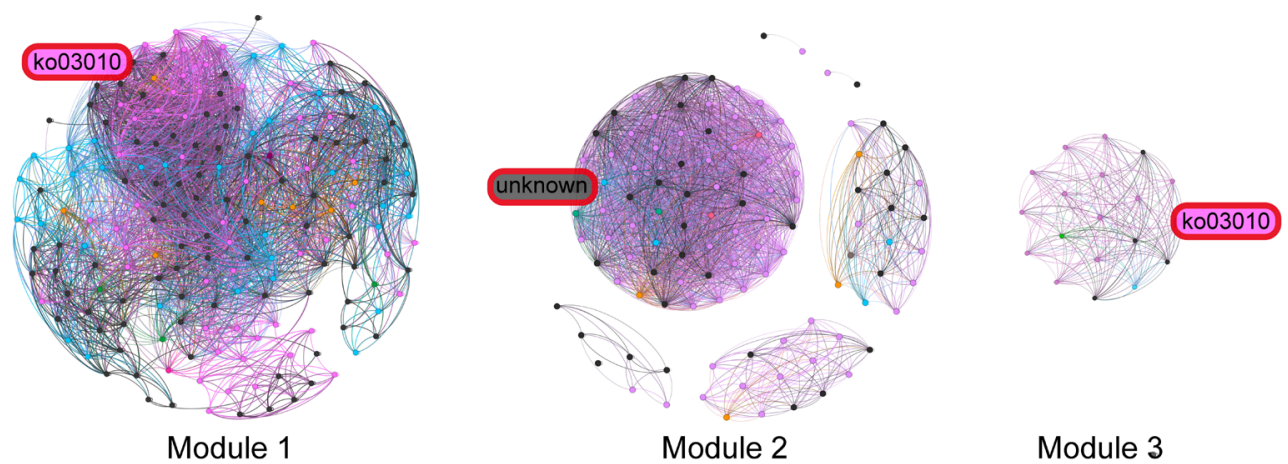

Module 1

Module 2

Module 3
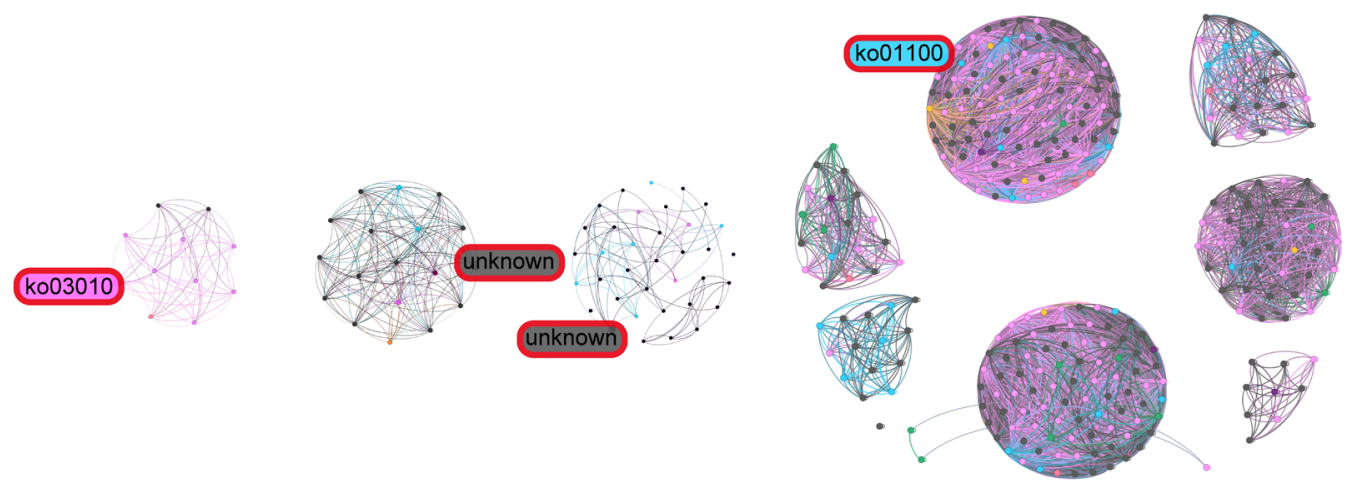

Module $4 \quad$ Module 5

Module 6

Module 7

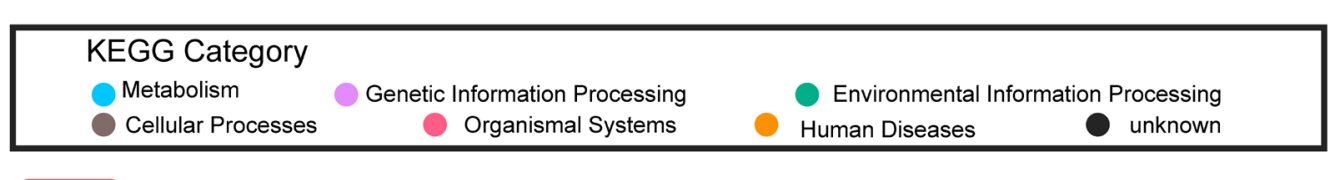

Hubba gene

Fig. 7. Gene co-expression networks were constructed using $W G C N A$. The function of each gene was annotated using the $K E G G$ database with labels arranged at the bottom. The genes marked with red borders are the hubba genes of each module. The serial number in the border represents the $K E G G$ pathway to which these genes belong.

$K E G G$ database, more than $53 \%$ of genes were assigned to at least one function. Three hubba genes of networks were annotated in pathway ko03010, one was annotated in ko01100, while the others could not be assigned to any pathways. In total, 19 genes targeting $K E G G$ pathways ko00195 and ko00196 were found, of which $79 \%$ occurred in module 1 .

\section{Discussion}

Photosynthetic capacity increased but efficiency decreased in high LI: The power values of growth rate, net photosynthetic rate, and CA activity were less than 0 , indicating the appearance of their predictable maximums with the increase in LI in the power curve model. This indicated that $0.46 \mathrm{~d}^{-1}, 2.45 \mathrm{nmol} \mathrm{L} \mathrm{s}^{-1} \mathrm{~s}^{-1}$, and $0.60 \times 10^{-1}$ EU $\mathrm{ml}^{-1}$ were probably their upper limits, respectively, even when the LI increased beyond $\mathrm{H}$. Comparatively, the power values of $\mathrm{Chl} a, \mathrm{PE}, \mathrm{PC}$, and AP content were between 0 and 1 , and hence these parameters continuously increased with LI, although the increase rate might be slow. CA plays a key role in catalyzing the critical first step of $\mathrm{C}_{4}$ photosynthesis and the hydration of $\mathrm{CO}_{2}$ to bicarbonate (Jensen et al. 2019), and the value of net photosynthetic rate reflected the ability to produce oxygen. The continuous increase in photosynthetic pigment content with stagnation in the abilities to absorb carbon dioxide and produce oxygen indicated that the photosynthetic efficiency per unit pigments might decrease with a further increase in LI. Furthermore, the content of pigmentproteins in the enrichment culture of Synechococcus assemblage might be lower than that of pure culture. The PC and APC contents of Synechococcus strain PCC 6715 (PT1) were determined; it showed that under continuous white light, the PC and APC contents were $8 \times 10^{-2}$ and 2 $\times 10^{-2} \mathrm{mg} \mathrm{mL}^{-1}$, which were about eight times higher than our results (Klepacz-Smółka et al. 2020).

Alteration of Synechococcus assemblages from in situ water to laboratory culture: Enrichment culture is closer to the natural state of Synechococcus assemblages than pure culture. However, after cultivation, the change in population composition and relative abundance was still large. Phylogenetically, marine Synechococcus formed a well-defined clade termed cluster 5 , which was divided into three subclusters: S5.1, S5.2, and S5.3; each subcluster can 
be further divided into various clades (Fuller et al. 2003). Generally, Synechococcus S5.1 is the most abundant and widespread subcluster in the ocean worldwide; in particular, its clades I, II, III, and IV dominate most sea waters (Zwirglmaier et al. 2008). After enrichment culture, Synechococcus assemblages of B1-I and B2-I were dominant in clade IX of S5.1. This clade was also observed in the adjacent Yellow Sea and the East China Sea but is rarely observed in other areas of the sea (Choi and Noh 2009, Wang et al. 2021). S5.2, occupying more than $15 \%$ of the site, is globally distributed in estuaries and brackish waters (Jing et al. 2009). The abundance of S5.3 is usually low (Ahlgren and Rocap 2012), and it was almost twice as much as S5.2 in this study. It is not clear whether this phenomenon is due to the unique niche occupation pattern of Synechococcus formed by the conditions of the semi-enclosed Bohai Sea. For further exploration, samples should be collected and cultured from more stations and different depths in the future research. Interestingly, the original dominant assemblages, including clade IX, S5.2, and S5.3, almost disappeared after enrichment culture. On the contrary, clade VIII, which accounts for less than $1 \%$ of the population in situ, was found to be the main population. Clade VIII is not a common Synechococcus lineage. Physiologically, some strains of clade VIII lack $\mathrm{PE}$ and cannot grow on nitrate (Fuller et al. 2003), and its distribution may be related to salinity, as it is found in hypersaline pools (Huang et al. 2012).

Synechococcus strains possess rich phenotypic diversity (Everroad and Wood 2012). In particular, the differences in evolutionary history between the major light-harvesting pigment PE and the core genome results in diverse PTs (Grébert et al. 2018). Some lineages are preferentially associated with a specific PT, such as most strains in clades I-IV and CRD1, while others exhibit a wide range of adaptability of PTs. As mentioned above, clade VIII normally lacks PE and is considered as PT1 (Fuller et al. 2003). However, according to the sequencing result based on the cpeBA operon, Synechococcus PT2 containing both PC and PE-I is the spectral phenotype corresponding to clade VIII. This can be attributed to the changing LI of the cultivating environment, which represents a selective agent that promotes the evolution of phenotypes adapted to different light environments, probably via mutation, positive directional selection, and horizontal gene transfer (Stomp et al. 2004).

Upregulation of genes related to photosynthesis: Metatranscriptomics is a powerful tool for capturing gene expression patterns in microbial communities without previous assumptions regarding the ongoing activities or dominant taxa (Frias-Lopez et al. 2008). Metatranscriptome research is still limited by difficulties in the construction of gene sets. In cases of prokaryotic polycistronic structure and low abundance of some genes, the lengths of the contigs are not sufficient for assembly, resulting in mapping that is far from satisfactory. In addition, deviations in results from the transcriptional data can be resolved only for individual genes with extremely high expression. To circumvent the above limitations, a mixed sample was used for metagenomic sequencing to obtain more comprehensive and accurate DNA-based metagenomic sequencing (Gilbert et al. 2008, Poretsky et al. 2010). The elimination of symbiotic species in the culture experiment of Synechococcus, even in the pure culture, is difficult (Zheng et al. 2020). Hence, the result of metatranscriptomic analysis may contain genes from these symbiotic species. However, Synechococcus is the only photoautotrophic organism in the culture system; hence, we believed that the regulation of genes related to photosynthesis was mostly because of the Synechococcus assemblage. Among the 7,145 differentially expressed genes, the downregulated genes occupied about $74 \%$ of the total, which is more than the proportion of upregulated genes. But interestingly, after annotation using the $K E G G$ database, all matched genes were significantly upregulated in pathway map ko00195 and ko00196 associated with the photosynthetic system, except for genes encoding F-type ATPase. Proteins or subunits of F-type ATPase are associated with the hydrolysis of a nucleoside triphosphate and electron transport and are also enriched in other pathways overlapping with ko00190 and ko01100 (Kanazawa et al. 1981). Therefore, under high LI, the metabolic activities associated with photosynthesis were enhanced in Synechococcus assemblages. This may inhibit other metabolic activities, although whether these repressed metabolic activities are due to Synechococcus or other symbiotic species remains unknown. Upregulation of genes related to photosynthesis did not necessarily correlate with the increase in photosynthetic efficiency. The interactions between gene expression and physiological parameters showed a negative correlation. A gene encoding the photosystem II protein, $P s b 27$, was upregulated, which is a key protein for efficient repair of photodamaged PSII (Chen et al. 2006). An increase in its expression indicated that the PSII had already undergone a certain extent of damage under the LI of $\mathrm{H}\left[243 \mu \mathrm{mol}\right.$ (photon) $\left.\mathrm{m}^{-2} \mathrm{~s}^{-1}\right]$.

Synergistic effect of population succession and gene regulation in photosynthetic adaptation: Population succession in Synechococcus assemblage is involved in its light adaptation, which was largely neglected in studies using pure culture. At the OTU level, both the phylogenetic and phenotypic composition of Synechococcus assemblage varied with the LI. In particular, in terms of phenotype, significant differences in the proportions of PTs between samples cultured under different LI were detected. Although RDA cannot be used to investigate the interaction between Synechococcus phenotypic composition and physiological parameters, Spearman correlations were observed between them. It is noteworthy that in addition to OTUs belonging to different PT, the relationship between OTUs belonging to the same PT and physiological parameters may differ, and may even be opposite. Therefore, the use of only one strain for studying physiological characteristics will lead to overgeneralization. Moreover, the relationship between the expression of some genes and physiological parameters, as well as some OTUs, demonstrated that gene regulation 
is also an important part of adaptation. Population succession and gene regulation are jointly responsible for photosynthetic adaptation with LI change. Compared to our results, photosynthetic parameters of purified Synechococcus isolates appear to vary more strongly with LI change in culture experiments in previous studies (Jodłowska and Śliwińska 2014, Śliwińska-Wilczewska et al. 2020). Such differences in the variation trends might be because of the specific characteristics of the selected isolates in purified experiments. In the screening of Synechococcus isolate, the adaptation from natural light to culture light is the key process, which may result in a similar response to LI among different strains (Nowack et al. 2015). However, changes in community diversity and self-regulation in population structure in enrichment experiments lead to influences from the environment that tend to be moderating and steady. Whether there is an effect of the quorum sensing in assemblages needs further experiments.

Potential genes involved in adapted regulation of light intensity: The enormity and complexity of the metatranscriptomic data render exploration of relationships between phenotypes and genes difficult. In fact, in addition to the two known pathways (ko00195 and ko00196), numerous other genes are involved in the regulation. WGCNA revealed that 678 expressed genes were transformed into seven co-expression modules. Among them, module 1 collected more than $79 \%$ of genes annotated in KEGG pathways ko00195 and ko00196. In total, $52.7 \%$ of genes in module 1 could be annotated using the $K E G G$ database, of which $56.3 \%$ belonged to ko03010 (ribosome). The hubba gene of module 1, RP-L4, encoding the large subunit ribosomal protein L4, was also in this pathway, which indicated that the regulation in response to light intensity activated the translation of various proteins. Previous proteomics research has shown that regulation of Synechococcus proteins involved in photosynthetic electron transport, carbon fixation, oxidative stress protection, and iron and nitrogen metabolism varied among Synechococcus strains that responded to an increase in LI (Mackey et al. 2017). rpoC1, a single copy housekeeping gene targeting the DNA-directed RNA polymerase beta subunit, is used to study the phylogenetic diversity of Synechococcus (Mühling et al. 2006). Consistent with the results of genomic sequencing, rpoC1 was not differentially expressed. Furthermore, other genes encoding the RNA polymerase subunits alpha $(r p o A)$ and beta $(r p o B)$ were found to be downregulated. The key function of rpoA is RNA polymerase assembly (Lee et al. 2012), while $r p o B$ and rpoC1 may collaborate to bind DNA and RNA or synthesize RNA (Sutherland and Murakami 2018). Therefore, it is reasonable to speculate that certain changes occur in the process of transcribing the genetic information stored in DNA to RNA under a gradient of light intensity. In addition, many upregulated genes were clustered in module 1 , the functions of which were associated with metabolic pathways (such as bsmB, ndhC, and gsmt), biosynthesis of secondary metabolites (chlL and pbsAl), carbon metabolism (aceA and $p r c L$ ), and $\mathrm{ABC}$ transporters (pstB). The functions of these genes could be closely interconnected to regulate adaptation under the stress of fluctuating light intensity.

Conclusions: The characteristics of Synechococcus assemblage in terms of physiology, genomics, and metatranscriptomics were revealed in the LI simulation experiment. We found that: (1) Physiological parameters exhibited positive responses to the LI in the studied scale. However, the model of the power curve predicted that the growth rate, net photosynthetic rate, and CA activity would reach their maximum value, while the contents of Chl $a$, PE, PC, and AP would keep increasing with further increase in LI beyond $243 \mu \mathrm{mol}$ (photon) $\mathrm{m}^{-2} \mathrm{~s}^{-1}$. (2) Compositions of Synechococcus assemblages derived from rpoC1 and cpeBA operon sequences shifted with LI change. Synechococcus PT2 was the most affected genotype. Such genomic succession could explain variations in physiological parameters, especially in the content of pigment proteins. (3) $74 \%$ of genes in metatranscriptome were downregulated in the $\mathrm{H}$ group. However, genes related to photosynthesis, most annotated in two KEGG pathways of ko00195 and ko00196, were commonly upregulated. Regulation of these genes was closely associated with the succession of genotypes and physiological shifts.

Based on the results, it is reasonable to infer that Synechococcus transfers energy to photosynthesis-related genes by reducing the expression of other metabolic activity-related genes, increasing photosynthetic parameters under high light intensity. Self-regulation in Synechococcus assemblages may lead to a moderate and steady response to the light intensity. However, whether there is an effect of the quorum sensing needs further experiments. Further studies will focus on investigating the expression and function of related genes using gene silencing assays to reveal the complete and accurate regulation strategies under conditions of varying LI. Mesoand large-scale field experiments should be conducted to better simulate the in situ Synechococcus assemblages and their adaptive strategies should be discussed.

\section{References}

Ahlgren N.A., Rocap G.: Culture isolation and cultureindependent clone libraries reveal new marine Synechococcus ecotypes with distinctive light and $\mathrm{N}$ physiologies. - Appl. Environ. Microb. 72: 7193-7204, 2006.

Ahlgren N.A., Rocap G.: Diversity and distribution of marine Synechococcus: multiple gene phylogenies for consensus classification and development of qPCR assays for sensitive measurement of clades in the ocean. - Front. Microbiol. 3: $213,2012$.

Altschul S.F., Madden T.L., Schäffer A.A. et al.: Gapped BLAST and PSI-BLAST: a new generation of protein database search programs. - Nucleic Acids Res. 25: 3389-3402, 1997.

Bolger A.M., Lohse M., Usadel B.: Trimmomatic: a flexible trimmer for Illumina sequence data. - Bioinformatics 30: 2114-2120, 2014.

Caporaso J.G., Kuczynski J., Stombaugh J. et al.: QIIME allows 
analysis of high-throughput community sequencing data. Nat. Methods 7: 335-336, 2010.

Chen H., Zhang D., Guo J. et al.: A Psb27 homologue in Arabidopsis thaliana is required for efficient repair of photodamaged photosystem II. - Plant Mol. Biol. 61: 567$575,2006$.

Chin C.H., Chen S.H., Wu H.H. et al.: cytoHubba: identifying hub objects and sub-networks from complex interactome. BMC Syst. Biol. 8: S11, 2014.

Choi D.H., Noh J.H.: Phylogenetic diversity of Synechococcus strains isolated from the East China Sea and the East Sea. FEMS Microbiol. Ecol. 69: 439-448, 2009.

de Mendiburu F.: Agricolae: statistical procedures for agricultural research. R package version 1, 2014.

Deng L., Ignacio-Espinoza J.C., Gregory A.C. et al.: Viral tagging reveals discrete populations in Synechococcus viral genome sequence space. - Nature 513: 242-245, 2014.

DuRand M.D., Olson R.J., Chisholm S.W.: Phytoplankton population dynamics at the Bermuda Atlantic Time-series station in the Sargasso Sea. - Deep Sea Res. Part II Top. Stud. Oceanogr. 48: 1983-2003, 2001.

Everroad R.C., Wood A.M.: Phycoerythrin evolution and diversification of spectral phenotype in marine Synechococcus and related picocyanobacteria. - Mol. Phylogenet. Evol. 64: 381-392, 2012.

Flombaum P., Gallegos J.L., Gordillo R.A. et al.: Present and future global distributions of the marine Cyanobacteria Prochlorococcus and Synechococcus. - P. Natl. Acad. Sci. USA 110: 9824-9829, 2013

Frias-Lopez J., Shi Y., Tyson G.W. et al.: Microbial community gene expression in ocean surface waters. - P. Natl. Acad. Sci. USA 105: 3805-3810, 2008.

Fu L., Niu B., Zhu Z. et al.: CD-HIT: accelerated for clustering the next-generation sequencing data. - Bioinformatics 28: 3150-3152, 2012.

Fuller N.J., Marie D., Partensky F. et al.: Clade-specific 16S ribosomal DNA oligonucleotides reveal the predominance of a single marine Synechococcus clade throughout a stratified water column in the Red Sea. - Appl. Environ. Microb. 69: 2430-2443, 2003.

Gao X., Chen C.T.A.: Heavy metal pollution status in surface sediments of the coastal Bohai Bay. - Water Res. 46: 19011911, 2012.

Gilbert J.A., Field D., Huang Y. et al.: Detection of large numbers of novel sequences in the metatranscriptomes of complex marine microbial communities. - PLoS ONE 3: e3042, 2008.

Grébert T., Doré H., Partensky F. et al.: Light color acclimation is a key process in the global ocean distribution of Synechococcus cyanobacteria. - P. Natl. Acad. Sci. USA 115: E2010-E2019, 2018.

Guidi L., Chaffron S., Bittner L. et al.: Plankton networks driving carbon export in the oligotrophic ocean. - Nature 532: 465470, 2016.

Halsey K.H., Jones B.M.: Phytoplankton strategies for photosynthetic energy allocation. - Annu. Rev. Mar. Sci. 7: 265-297, 2015.

Hu H., Boisson-Dernier A., Israelsson-Nordström M. et al.: Carbonic anhydrases are upstream regulators of $\mathrm{CO}_{2-}^{-}$ controlled stomatal movements in guard cells. - Nat. Cell Biol. 12: 87-93, 2010.

Huang S., Wilhelm S.W., Harvey H.R. et al.: Novel lineages of Prochlorococcus and Synechococcus in the global oceans. ISME J. 6: 285-297, 2012.

Hunter-Cevera K.R., Post A.F., Peacock E.E., Sosik H.M.: Diversity of Synechococcus at the Martha's Vineyard coastal observatory: Insights from culture isolations, clone libraries, and flow cytometry. - Microb. Ecol. 71: 276-289, 2016.

Hyatt D., LoCascio P.F., Hauser L.J., Uberbacher E.C.: Gene and translation initiation site prediction in metagenomic sequences. - Bioinformatics 28: 2223-2230, 2012.

Jensen E.L., Clement R., Kosta A. et al:: A new widespread subclass of carbonic anhydrase in marine phytoplankton. ISME J. 13: 2094-2106, 2019.

Jing H., Liu H., Suzuki K.: Phylogenetic diversity of marine Synechococcus spp. in the Sea of Okhotsk. - Aquat. Microb. Ecol. 56: 55-63, 2009.

Jodłowska S., Śliwińska S.: Effects of light intensity and temperature on the photosynthetic irradiance response curves and chlorophyll fluorescence in three picocyanobacterial strains of Synechococcus. - Photosynthetica 52: 223-232, 2014.

Kanazawa H., Kayano T., Mabuchi K., Futai M.: Nucleotide sequence of the genes coding for $\alpha, \beta$ and $\gamma$ subunits of the proton-translocating ATPase of Escherichia coli. - Biochem. Bioph. Res. Co. 103: 604-612, 1981.

Kanehisa M., Goto S.: KEGG: Kyoto encyclopedia of genes and genomes. - Nucleic Acids Res. 28: 27-30, 2000.

Klepacz-Smółka A., Pietrzyk D., Szeląg R. et al.: Effect of light colour and photoperiod on biomass growth and phycocyanin production by Synechococcus PCC 6715. - Bioresource Technol. 313: 123700, 2020.

Klopfenstein D.V., Zhang L., Pedersen B.S. et al.: GOATOOLS: A Python library for Gene Ontology analyses. - Sci Rep.-UK 8: $10872,2018$.

Kopylova E., Noé L., Touzet H.: SortMeRNA: fast and accurate filtering of ribosomal RNAs in metatranscriptomic data. Bioinformatics 28: 3211-3217, 2012.

Langfelder P., Horvath S.: WGCNA: an R package for weighted correlation network analysis. - BMC Bioinformatics 9: 559, 2008.

Lee D.J., Minchin S.D., Busby S.J.W.: Activating transcription in bacteria. - Annu. Rev. Microbiol. 66: 125-152, 2012.

Li B., Dewey C.N.: RSEM: accurate transcript quantification from RNA-Seq data with or without a reference genome. BMC Bioinformatics 12: 323, 2011.

Li D., Luo R., Liu C.M. et al.: MEGAHIT v1.0: A fast and scalable metagenome assembler driven by advanced methodologies and community practices. - Methods 102: 3-11, 2016.

Li J., Wang T., Yu S. et al.: Community characteristics and ecological roles of bacterial biofilms associated with various algal settlements on coastal reefs. - J. Environ. Manage. 250: $109459,2019$.

Lindell D., Post A.F.: Ultraphytoplankton succession is triggered by deep winter mixing in the Gulf of Aqaba (Eilat), Red Sea. - Limnol. Oceanogr. 40: 1130-1141, 1995.

Liu X., Liu D., Wang Y. et al.: Temporal and spatial variations and impact factors of nutrients in Bohai Bay, China. - Mar. Pollut. Bull. 140: 549-562, 2019.

Mackey K.R.M., Paytan A., Grossman A.R., Bailey S.: A photosynthetic strategy for coping in a high-light, low-nutrient environment. - Limnol. Oceanogr. 53: 900-913, 2008.

Mackey K.R.M., Post A.F., McIlvin M.R., Saito M.A.: Physiological and proteomic characterization of light adaptations in marine Synechococcus. - Environ. Microbiol. 19: 2348-2365, 2017.

Mei W., Yu G., Lai J. et al.: basicTrendline: Add trendline and confidence interval of basic regression models to plot. R package version 2, 2018.

Mittal R., Tavanandi H.A., Mantri V.A., Raghavarao K.S.M.S.: Ultrasound assisted methods for enhanced extraction of phycobiliproteins from marine macro-algae, Gelidium pusillum (Rhodophyta). - Ultrason. Sonochem. 38: 92-103, 
2017.

Mühling M., Fuller N.J., Somerfield P.J. et al.: High resolution genetic diversity studies of marine Synechococcus isolates using rpoCl-based restriction fragment length polymorphism. - Aquat. Microb. Ecol. 45: 263-275, 2006.

Nowack S., Olsen M.T., Schaible G.A. et al.: The molecular dimension of microbial species: 2. Synechococcus strains representative of putative ecotypes inhabiting different depths in the Mushroom Spring microbial mat exhibit different adaptive and acclimative responses to light. - Front. Microbiol. 6: 626, 2015.

Oksanen J., Blanchet F.G., Kindt R. et al.: Package 'vegan'. Community ecology package, version 2, 2013.

Paulsen M.L., Doré H., Garczarek L. et al.: Synechococcus in the Atlantic gateway to the Arctic Ocean. - Front. Mar. Sci. 3: 191, 2016.

Poretsky R.S., Sun S., Mou X., Moran M.A.: Transporter genes expressed by coastal bacterioplankton in response to dissolved organic carbon. - Environ. Microbiol. 12: 616-627, 2010.

Roberts A., Pachter L.: Streaming fragment assignment for realtime analysis of sequencing experiments. - Nat. Methods 10: 71-73, 2013

Robinson M.D., McCarthy D.J., Smyth G.K.: edgeR: a Bioconductor package for differential expression analysis of digital gene expression data. - Bioinformatics 26: 139-140, 2010.

Rocap G., Larimer F.W., Lamerdin J. et al.: Genome divergence in two Prochlorococcus ecotypes reflects oceanic niche differentiation. - Nature 424: 1042-1047, 2003.

Scanlan D.J., Ostrowski M., Mazard S. et al.: Ecological genomics of marine picocyanobacteria. - Microbiol. Mol. Biol. Rev. 73: 249-299, 2009.

Śliwińska-Wilczewska S., Konarzewska Z., Wiśniewska K., Konik M.: Photosynthetic pigments changes of three phenotypes of picocyanobacteria Synechococcus sp. under different light and temperature conditions. - Cells 9: 2030, 2020.

Sohm J.A., Ahlgren N.A., Thomson Z.J. et al.: Co-occurring Synechococcus ecotypes occupy four major oceanic regimes defined by temperature, macronutrients and iron. - ISME J. 10: 333-345, 2016.

Stomp M., Huisman J., De Jongh F. et al.: Adaptive divergence in pigment composition promotes phytoplankton biodiversity. Nature 432: 104-107, 2004.

Sutherland C., Murakami K.S.: An introduction to the structure and function of the catalytic core enzyme of Escherichia coli RNA polymerase. - EcoSal Plus 8: 10.1128/ecosalplus.ESP0004-2018, 2018.

Ueno Y., Shimakawa G., Aikawa S. et al.: Photoprotection mechanisms under different $\mathrm{CO}_{2}$ regimes during photosynthesis in a green alga Chlorella variabilis. - Photosynth. Res. 144: 397-407, 2020.

van der Hout C.M., Witbaard R., Bergman M.J.N. et al.: The dynamics of suspended particulate matter (SPM) and chlorophyll- $a$ from intratidal to annual time scales in a coastal turbidity maximum. - J. Sea Res. 127: 105-118, 2017.

Wagner H., Jakob T., Lavaud J., Wilhelm C.: Photosystem II cycle activity and alternative electron transport in the diatom Phaeodactylum tricornutum under dynamic light conditions and nitrogen limitation. - Photosynth. Res. 128: 151-161, 2016.

Wang T., Chen X., Qin S., Li J.: Phylogenetic and phenogenetic diversity of Synechococcus along a Yellow Sea section reveal its environmental dependent distribution and co-occurrence microbial pattern. - J. Mar. Sci. Eng. 9: 1018, 2021.

Xia X., Cheung S., Endo H. et al.: Latitudinal and vertical variation of Synechococcus assemblage composition along $170^{\circ} \mathrm{W}$ transect from the South Pacific to the Arctic Ocean. Microb. Ecol. 77: 333-342, 2019.

Xia X., Liu H., Choi D., Noh J.H.: Variation of Synechococcus pigment genetic diversity along two turbidity gradients in the China Seas. - Microb. Ecol. 75: 10-21, 2018.

Zheng Q., Wang Y., Lu J. et al.: Metagenomic and metaproteomic insights into photoautotrophic and heterotrophic interactions in a Synechococcus culture. - mBio 11: e03261-19, 2020.

Zohdi E., Abbaspour M.: Harmful algal blooms (red tide): a review of causes, impacts and approaches to monitoring and prediction. - Int. J. Environ. Sci. Technol. 16: 1789-1806, 2019.

Zwirglmaier K., Jardillier L., Ostrowski M. et al.: Global phylogeography of marine Synechococcus and Prochlorococcus reveals a distinct partitioning of lineages among oceanic biomes. - Environ. Microbiol. 10: 147-161, 2008.

(C) The authors. This is an open access article distributed under the terms of the Creative Commons BY-NC-ND Licence. 